\title{
The impact of future emission policies on tropospheric ozone using a pa- rameterised approach
}

Steven T. Turnock et al.

Correspondence to: Steven T. Turnock (steven.turnock@ metoffice.gov.uk)

The copyright of individual parts of the supplement might differ from the CC BY 4.0 License. 
S1 Schematic of the parameterisation

\section{Calculate fractional emission change $(r)$}

$$
r_{i j}=\frac{\Delta E_{i j}}{-0.2 \times E_{i j}}
$$

\section{Generate scaling factors (f)}

2.1. Linear scaling of $\mathrm{O}_{3}$ response

$$
f_{i j}=r_{i j}
$$

2.2. Non-linear scaling accounting for reduced $\mathrm{O}_{3}$ increases from $\mathrm{NOx}$ and $\mathrm{CH}_{4}$

$$
f_{i j}=0.95 r_{i j}+0.05 r_{i j}^{2}
$$

2.3. Non-linear scaling for titration regimes where decreasing NOx increases $\mathrm{O}_{3}$

$$
f_{i j}=1.05 r_{i j}-0.05 r_{i j}^{2}
$$

\section{Apply different scaling factors to precursors and source regions under different chemical regimes}

\section{$\mathrm{O}_{3}$ Production Regime \\ Precursor Perturbation: \\ $\mathrm{CO}$ and NMVOCs - Linear scaling (1) \\ NOx and $\mathrm{CH}_{4}-$ Non-linear scaling (2)}

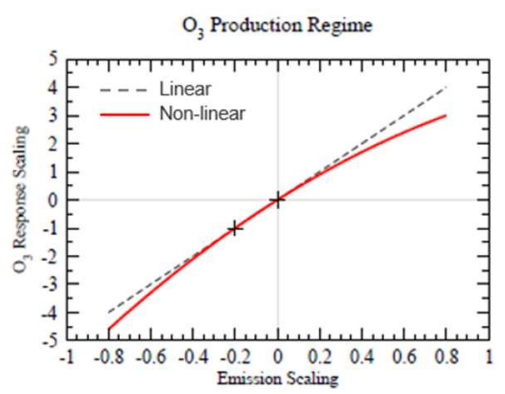

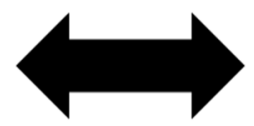

\section{$\mathrm{O}_{3}$ Titration Regime}

Precursor Perturbation:

Increased NOx - Linear scaling (1)

Decreased NOx - Non-linear scaling (3)

$\mathrm{O}_{3}$ Titration Regime

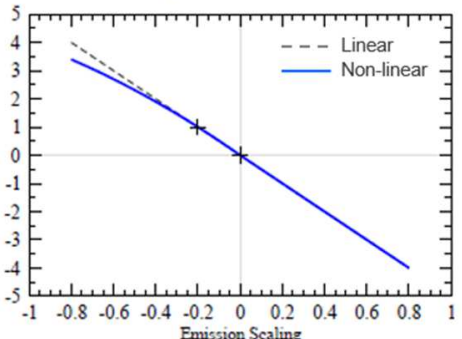

Figure S1: Schematic showing the different steps used in the parameterisation from calculating a fractional emission change (1), to generating an emission scaling factor (2) and applying this to the appropriate precursor in a particular chemical regime (3). The figures at the bottom illustrate the effect of applying the quadratic function compared to the linear one in the different chemical regimes. 
S2 Additional tests on the linear scaling used in the parameterisation based on multiple models as input
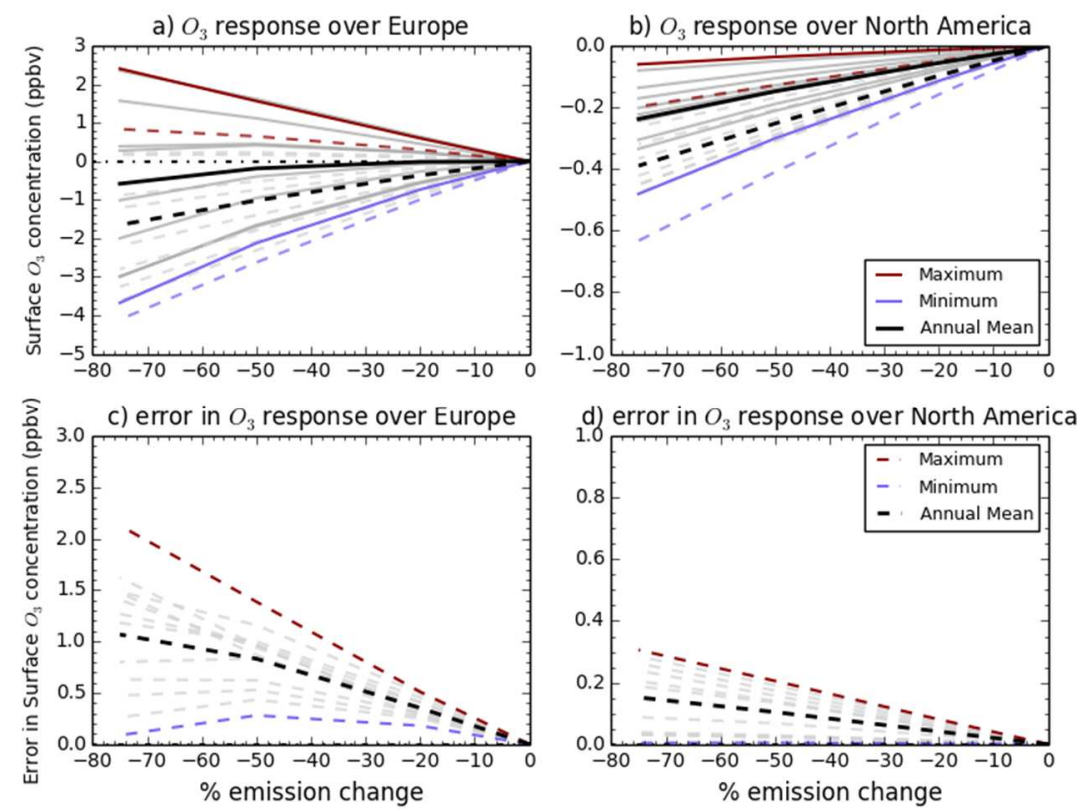

Figure S2: Sensitivity of monthly surface $\mathrm{O}_{3}$ changes in HadGEM2-ES (solid lines) and that of the parameterised response using solely multi-model response as input (dashed lines) to $20 \%, 50 \%$ and $75 \%$ reduction in all precursor emissions over the European source region (a) and a remote region receptor of North America (b). The difference between HadGEM2-ES and the parameterised response is shown over Europe (c) and North America (d). Annual mean values are in black with monthly responses in grey and the highest and lowest months highlighted in red and blue respectively. 
S3 Percentage change in CO and NMVOC precursor emissions for each of the ECLIPSE emission scenarios over the TF-HTAP2 regions and used with the parameterisation

Table S1. Percentage change in global and regional CO emissions relative to 2010 over each TF-HTAP2 region for the different ECLIPSE V5a emission scenarios (CLE, CLIM and MTFR). MTFR scenarios are only available for 2030 and 2050.

\begin{tabular}{lcccccccccc}
\hline TF-HTAP2 Region & \multicolumn{8}{c}{ Annual total emission change (\%) from 2010 } \\
& \multicolumn{9}{c}{ CLE } & \multicolumn{7}{c}{ CLIM } & \multicolumn{2}{c}{ MTFR } \\
& $\mathbf{2 0 2 0}$ & $\mathbf{2 0 3 0}$ & $\mathbf{2 0 4 0}$ & $\mathbf{2 0 5 0}$ & $\mathbf{2 0 2 0}$ & $\mathbf{2 0 3 0}$ & $\mathbf{2 0 4 0}$ & $\mathbf{2 0 5 0}$ & $\mathbf{2 0 3 0}$ & $\mathbf{2 0 5 0}$ \\
\hline Global CO & -7 & -14 & -10 & -8 & -12 & -22 & -23 & -25 & -64 & -70 \\
& \multicolumn{7}{c}{ Regional CO Emissions } \\
Central America & 0 & -17 & -14 & -6 & -16 & -38 & -41 & -43 & -59 & -76 \\
Central Asia & 42 & 42 & 35 & 32 & 44 & 18 & -7 & -23 & -45 & -82 \\
East Asia & -18 & -35 & -37 & -41 & -24 & -40 & -43 & -47 & -71 & -75 \\
Europe & -22 & -34 & -36 & -37 & -26 & -42 & -48 & -50 & -64 & -70 \\
Middle East & 21 & 16 & 42 & 66 & -12 & -23 & -28 & -29 & -32 & -40 \\
North Africa & -8 & -2 & 15 & 31 & -27 & -24 & -17 & -10 & -63 & -67 \\
North America & -11 & -27 & -29 & -31 & 9 & -23 & -29 & -32 & -52 & -70 \\
North Pole & -30 & -38 & -42 & -41 & -37 & -47 & -47 & -51 & -70 & -81 \\
Ocean & -2 & 10 & 16 & 23 & -13 & -10 & -12 & -12 & -51 & -61 \\
Pacific Aus NZ & -17 & -40 & -41 & -42 & -22 & -46 & -48 & -51 & -61 & -69 \\
Rus Bel Ukr & -10 & -4 & 0 & 5 & -15 & -13 & -7 & -6 & -57 & -60 \\
Southern Africa & 5 & 11 & 17 & 24 & 2 & 7 & 8 & 9 & -64 & -69 \\
South America & -7 & -7 & -1 & 6 & -9 & -11 & -6 & -1 & -58 & -59 \\
South Asia & 2 & 2 & 10 & 16 & -1 & -4 & -4 & -7 & -66 & -65 \\
South East Asia & -6 & -9 & 1 & 10 & -11 & -16 & -15 & -16 & -67 & -77 \\
\hline
\end{tabular}

Table S2. Percentage change in global and regional NMVOC emissions relative to 2010 over each TF-HTAP2 region for the different ECLIPSE V5a emission scenarios (CLE, CLIM and MTFR). MTFR scenarios are only available for 2030 and 2050.

\begin{tabular}{|c|c|c|c|c|c|c|c|c|c|c|}
\hline \multirow[t]{3}{*}{ TF-HTAP2 Region } & \multicolumn{10}{|c|}{ Annual total emission change (\%) from 2010} \\
\hline & \multicolumn{4}{|c|}{ CLE } & \multicolumn{4}{|c|}{ CLIM } & \multicolumn{2}{|c|}{ MTFR } \\
\hline & 2020 & 2030 & 2040 & 2050 & 2020 & 2030 & 2040 & 2050 & 2030 & 2050 \\
\hline Global NMVOC & -4 & -6 & 0 & 6 & -3 & -3 & -1 & -1 & -68 & -64 \\
\hline \multicolumn{11}{|c|}{ Regional NMVOC Emissions } \\
\hline Central America & 4 & -2 & 1 & 7 & -5 & -11 & -12 & -11 & -49 & -60 \\
\hline Central Asia & 46 & 53 & 50 & 50 & 51 & 39 & 23 & 12 & -18 & -49 \\
\hline East Asia & 2 & -6 & -4 & -2 & 1 & -5 & -4 & -5 & -54 & -61 \\
\hline Europe & -18 & -24 & -25 & -26 & -19 & -25 & -27 & -28 & -55 & -56 \\
\hline Middle East & 9 & 11 & 30 & 45 & -3 & -4 & 2 & 5 & -18 & -10 \\
\hline North Africa & -3 & -2 & 6 & 23 & -14 & -13 & -10 & 1 & -50 & -55 \\
\hline North America & -8 & -25 & -26 & -28 & 4 & -18 & -20 & -21 & -58 & -63 \\
\hline North Pole & -24 & -32 & -29 & -27 & -25 & -35 & -35 & -36 & -47 & -46 \\
\hline Ocean & 4 & 8 & 14 & 21 & -0.4 & 0.2 & 2 & 4 & -27 & -27 \\
\hline Pacific Aus NZ & -13 & -28 & -29 & -33 & -13 & -28 & -28 & -32 & -60 & -65 \\
\hline Rus Bel Ukr & -11 & -15 & -11 & -7 & -14 & -21 & -19 & -19 & -56 & -61 \\
\hline Southern Africa & 5 & 8 & 16 & 25 & 2 & 5 & 9 & 13 & -62 & -62 \\
\hline South America & 0 & 0 & 9 & 20 & 8 & 4 & 11 & 18 & -52 & -56 \\
\hline South Asia & 6 & 19 & 34 & 47 & 4 & 13 & 16 & 16 & -58 & -48 \\
\hline South East Asia & 0 & 3 & 19 & 33 & -15 & -19 & -16 & -14 & -56 & -64 \\
\hline
\end{tabular}


S4 Additional figures showing the $\mathrm{O}_{3}$ response from the parameterisation and HadGEM2-ES to emission changes in 2030 for the ECLIPSE CLE scenario
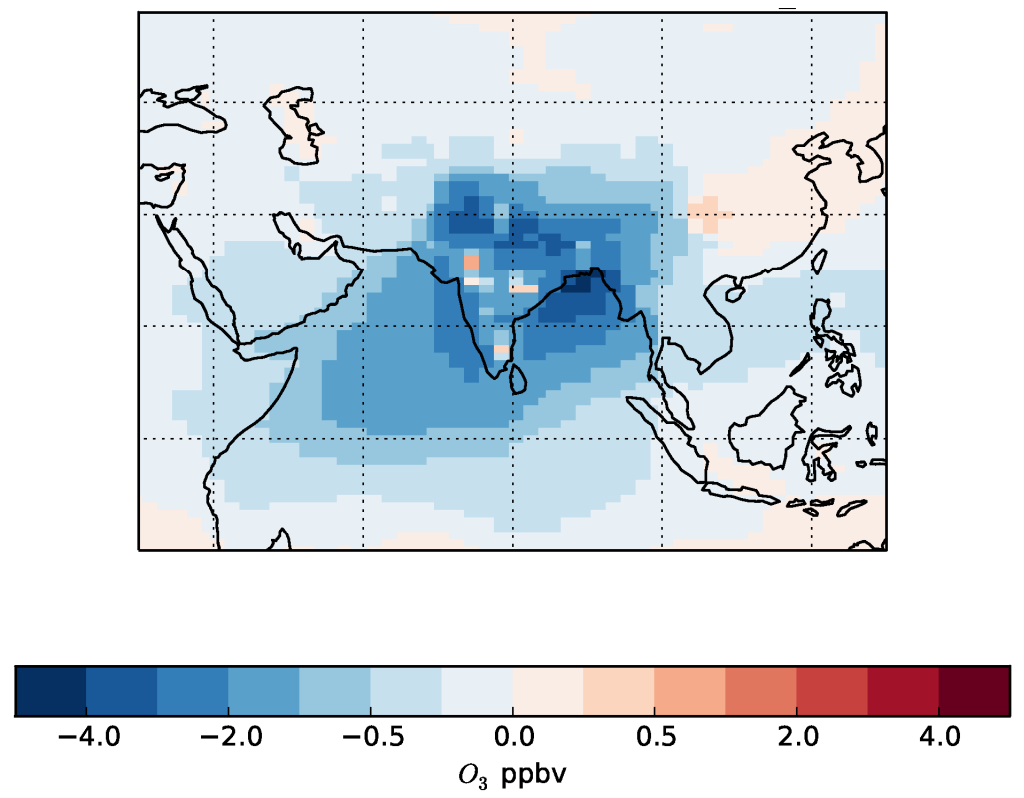

Figure S3: January ozone response in HadGEM2-ES to a $20 \%$ emission reduction over South Asia of all anthropogenic precursor emissions (NOx, CO, NMVOCs)
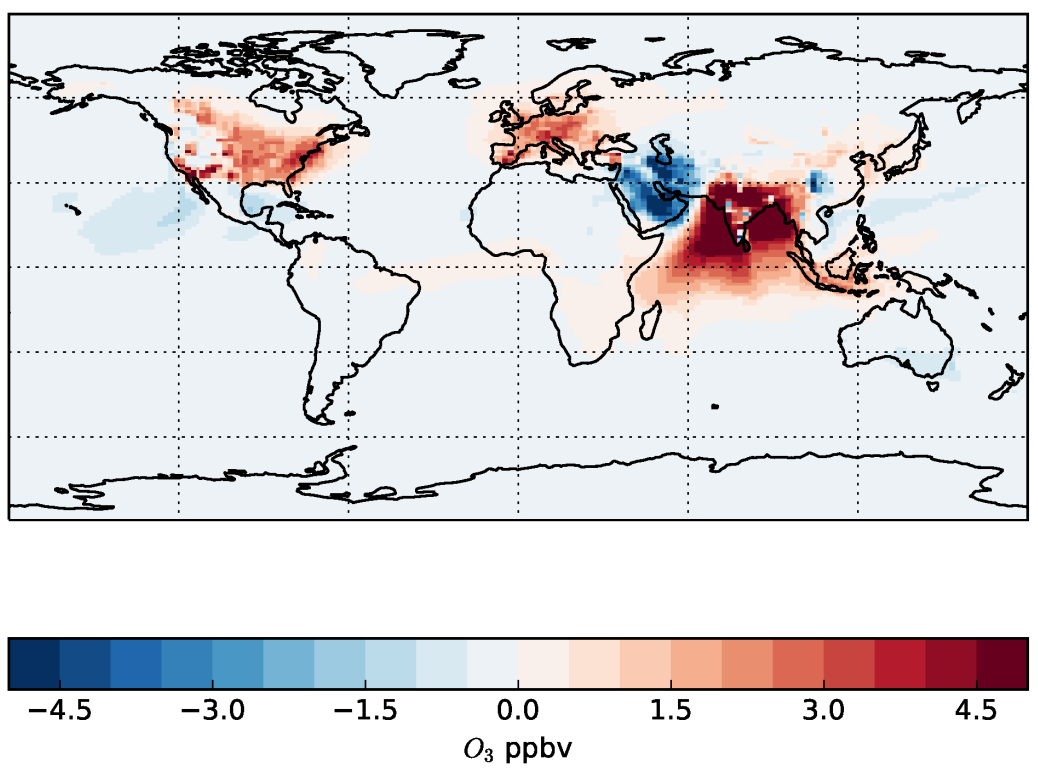

Figure S4: January ozone response from the parameterisation to the emission changes in the ECLIPSE CLE 2030 scenario, relative to 2010 

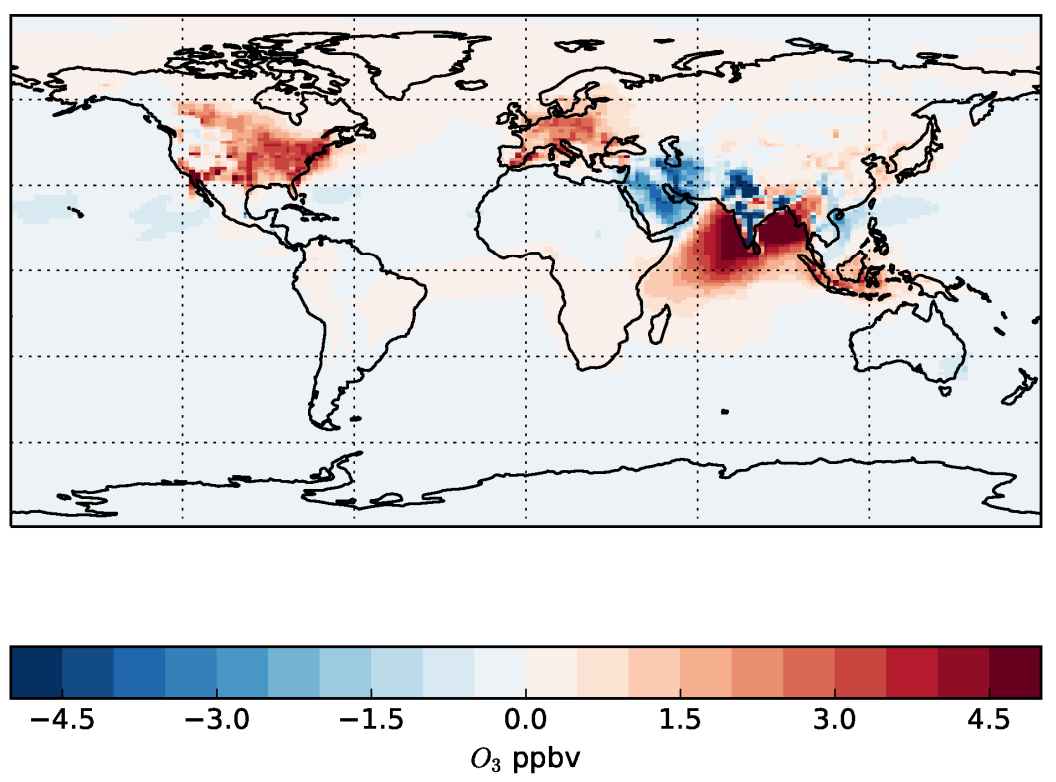

Figure S5: January ozone response from HadGEM2-ES to the emission changes within the ECLIPSE CLE 2030 scenario, relative to 2010 
S5 Additional regional results from the parameterisation using the CMIP5 emission scenarios in 2050

Table S3. Annual mean surface $\mathrm{O}_{3}$ response (ppbv plus one standard deviation) in 2050 (relative to 2010) using the parameterisation for each RCP scenario.

\begin{tabular}{lcccc}
\hline & \multicolumn{3}{c}{ Surface O3 response from 2010 to 2050 (ppbv) } \\
\hline TF-HTAP2 Region & RCP2.6 & RCP4.5 & RCP6.0 & RCP8.5 \\
\hline Central America & $-3.0+/-0.3$ & $-1.5+/-0.2$ & $-1.8+/-0.3$ & $+0.6+/-0.4$ \\
Central Asia & $-4.4+/-0.5$ & $-2.6+/-0.4$ & $-1.8+/-0.4$ & $+0.5+/-0.6$ \\
East Asia & $-4.7+/-0.3$ & $-2.9+/-0.3$ & $+0.2+/-0.2$ & $+0.4+/-0.3$ \\
Europe & $-4.4+/-0.3$ & $-2.9+/-0.2$ & $-1.6+/-0.2$ & $+0.3+/-0.1$ \\
Middle East & $-4.3+/-0.5$ & $-1.9+/-0.7$ & $-1.4+/-0.5$ & $+2.3+/-1.1$ \\
North Africa & $-4.0+/-0.4$ & $-2.1+/-0.3$ & $-1.5+/-0.3$ & $+0.9+/-0.7$ \\
North America & $-5.0+/-0.3$ & $-3.5+/-0.3$ & $-1.8+/-0.2$ & $-0.7+/-0.3$ \\
North Pole & $-3.3+/-0.5$ & $-2.1+/-0.4$ & $-0.8+/-0.3$ & $+0.1+/-0.6$ \\
Ocean & $-2.3+/-0.2$ & $-1.0+/-0.1$ & $-0.7+/-0.1$ & $+1.0+/-0.4$ \\
Pacific Aus NZ & $-1.4+/-0.6$ & $-0.3+/-0.5$ & $-0.4+/-0.4$ & $+1.4+/-0.6$ \\
Rus Bel Ukr & $-3.6+/-0.2$ & $-2.3+/-0.1$ & $-0.9+/-0.1$ & $+0.4+/-0.4$ \\
Southern Africa & $-0.8+/-0.2$ & $+0.3+/-0.1$ & $-0.3+/-0.1$ & $+2.1+/-0.5$ \\
South America & $-0.9+/-0.2$ & $-0.1+/-0.3$ & $-0.6+/-0.1$ & $+1.3+/-0.3$ \\
South Asia & $-0.8+/-0.2$ & $+2.4+/-0.2$ & $-0.5+/-0.1$ & $+4.0+/-0.4$ \\
South East Asia & $-1.3+/-0.2$ & $-0.2+/-0.2$ & $+0.1+/-0.1$ & $-0.4+/-0.5$ \\
South Pole & $-0.9+/-0.2$ & $-0.1+/-0.2$ & $-0.2+/-0.1$ & $+1.3+/-0.5$ \\
\hline
\end{tabular}


S6 Percentage change in CO and NMVOC precursor emissions for each of the CMIP6 SSPs over the TFHTAP2 regions and used with the parameterisation

Table S4. Percentage change in global and regional CO emissions relative to 2010 over each TF-HTAP2 region for the different CMIP6 emission scenarios (SSP3 BASE, SSP2 60 and SSP1 26)

\begin{tabular}{|c|c|c|c|c|c|c|c|c|c|c|c|c|}
\hline \multirow[t]{3}{*}{ TF-HTAP2 Region } & \multicolumn{12}{|c|}{ Annual total emission change (\%) from 2010} \\
\hline & \multicolumn{4}{|c|}{ SSP1 26} & \multicolumn{4}{|c|}{ SSP2 60} & \multicolumn{4}{|c|}{ SSP3 BASE } \\
\hline & 2020 & 2030 & 2040 & 2050 & 2020 & 2030 & 2040 & 2050 & 2020 & 2030 & 2040 & 2050 \\
\hline Global CO & -19 & -38 & -42 & -45 & -9 & -12 & -17 & -24 & 2 & 2 & 2 & 1 \\
\hline \multicolumn{13}{|c|}{ Regional CO Emissions } \\
\hline $\begin{array}{l}\text { Europe, North America, } \\
\text { Pacific Aus NZ } \\
\text { Central Asia, }\end{array}$ & -19 & -40 & -45 & -48 & -16 & -23 & -30 & -35 & -1 & -6 & -8 & -10 \\
\hline $\begin{array}{l}\text { Rus Bel Ukr } \\
\text { East Asia, South Asia, }\end{array}$ & 1 & 3 & -17 & -27 & -5 & -4 & -8 & -16 & 2 & 2 & 4 & 2 \\
\hline $\begin{array}{l}\text { South East Asia } \\
\text { Middle East, North }\end{array}$ & -23 & -42 & -46 & -51 & -12 & -16 & -27 & -39 & 7 & 10 & 12 & 10 \\
\hline $\begin{array}{l}\text { Africa, Southern Africa } \\
\text { Central America, }\end{array}$ & -15 & -32 & -39 & -36 & 0 & 3 & 7 & 8 & -2 & -5 & -6 & -7 \\
\hline South America & -18 & -38 & -37 & -44 & -14 & -24 & -30 & -38 & 1 & -2 & -1 & 0 \\
\hline
\end{tabular}

Table S5. Percentage change in global and regional NMVOC emissions relative to 2010 over each TF-HTAP2 region for the different CMIP6 emission scenarios (SSP3 BASE, SSP2 60 and SSP1 26)

\begin{tabular}{|c|c|c|c|c|c|c|c|c|c|c|c|c|}
\hline \multirow[t]{3}{*}{ TF-HTAP2 Region } & \multicolumn{12}{|c|}{ Annual total emission change (\%) from 2010} \\
\hline & \multicolumn{4}{|c|}{ SSP1 26} & \multicolumn{4}{|c|}{ SSP2 60} & \multicolumn{4}{|c|}{ SSP3 BASE } \\
\hline & 2020 & 2030 & 2040 & 2050 & 2020 & 2030 & 2040 & 2050 & 2020 & 2030 & 2040 & 2050 \\
\hline Global NMVOC & -21 & -33 & -32 & -28 & -6 & -9 & -13 & -17 & 3 & 3 & -6 & -7 \\
\hline \multicolumn{13}{|c|}{ Regional NMVOC Emissions } \\
\hline $\begin{array}{l}\text { Europe, North America, } \\
\text { Pacific Aus NZ } \\
\text { Central Asia, }\end{array}$ & -35 & -54 & -62 & -68 & -14 & -21 & -25 & -27 & -2 & -7 & -11 & -12 \\
\hline $\begin{array}{l}\text { Rus Bel Ukr } \\
\text { East Asia, South Asia, }\end{array}$ & -17 & -44 & -58 & -69 & -6 & -10 & -12 & -11 & 3 & 4 & -1 & -3 \\
\hline $\begin{array}{l}\text { South East Asia } \\
\text { Middle East, North }\end{array}$ & -26 & -35 & -41 & -49 & -6 & -9 & -15 & -22 & 7 & 11 & 11 & 10 \\
\hline $\begin{array}{l}\text { Africa, Southern Africa } \\
\text { Central America, }\end{array}$ & -10 & -18 & 6 & 46 & 0 & 3 & 3 & -2 & 1 & 3 & 5 & 6 \\
\hline South America & -21 & -41 & -40 & -47 & -14 & -24 & -25 & -30 & 3 & 4 & 2 & 4 \\
\hline
\end{tabular}


S7 Additional figures showing the source contribution analysis for the other TF-THAP2 source regions using the ECLIPSE emission scenarios in the parameterisation

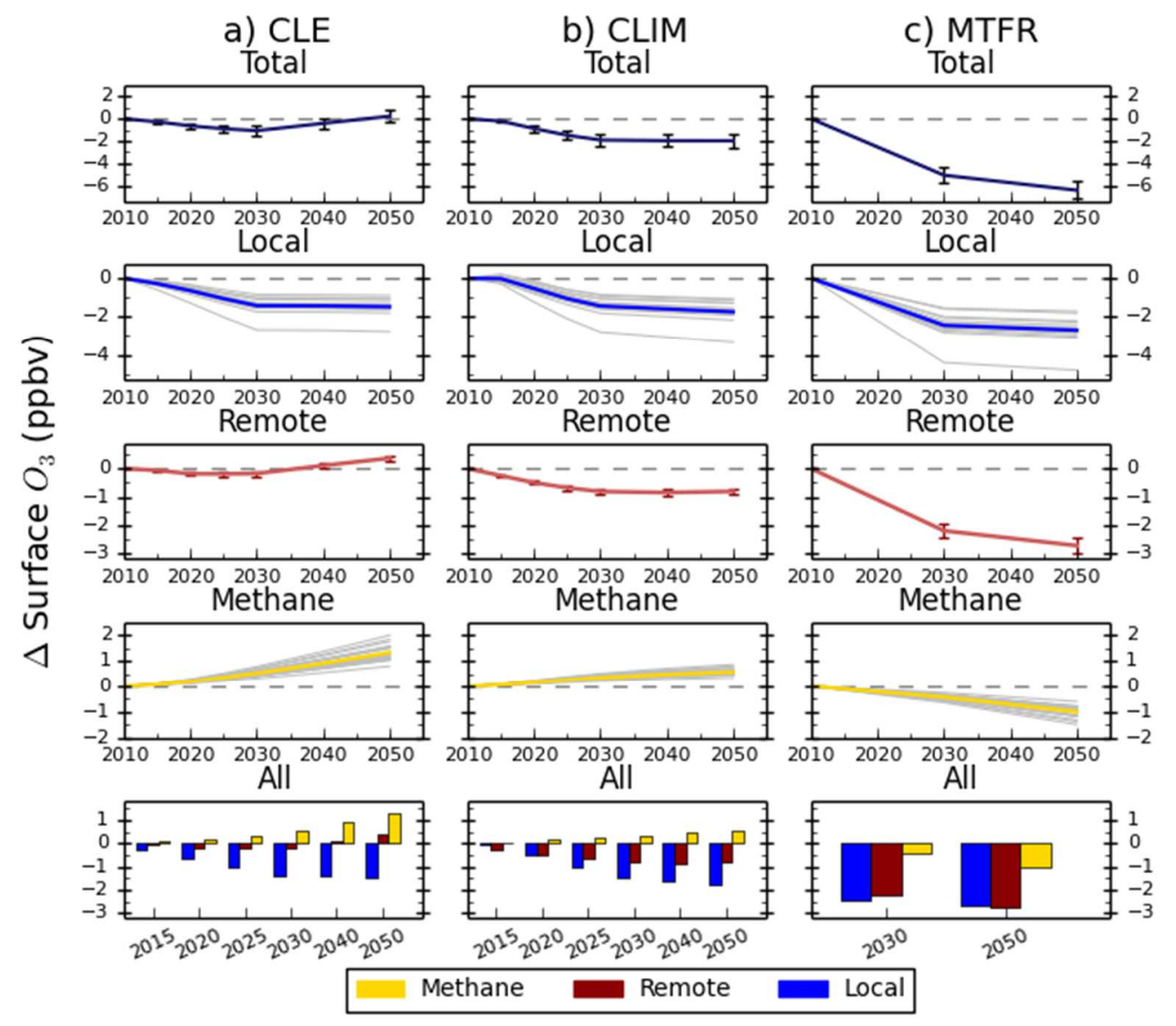

Figure S6: Total annual mean change in regional surface $\mathrm{O}_{3}$ concentrations over North America and the contribution of local (blue), remote (red) and methane (gold) sources between 2010 and 2050 from the parameterisation for the ECLIPSEv5a emissions under the CLE (a), CLIM (b) and MTFR (c) scenarios. Grey lines on the local and methane panels represent individual model estimates of $\mathrm{O}_{3}$ changes, showing the spread in model responses; Solid lines show the multi-model mean. Error bars represent one standard deviation over the model range. The last row of panels shows the $\mathrm{O}_{3}$ response from individual sources plotted together for each year. 


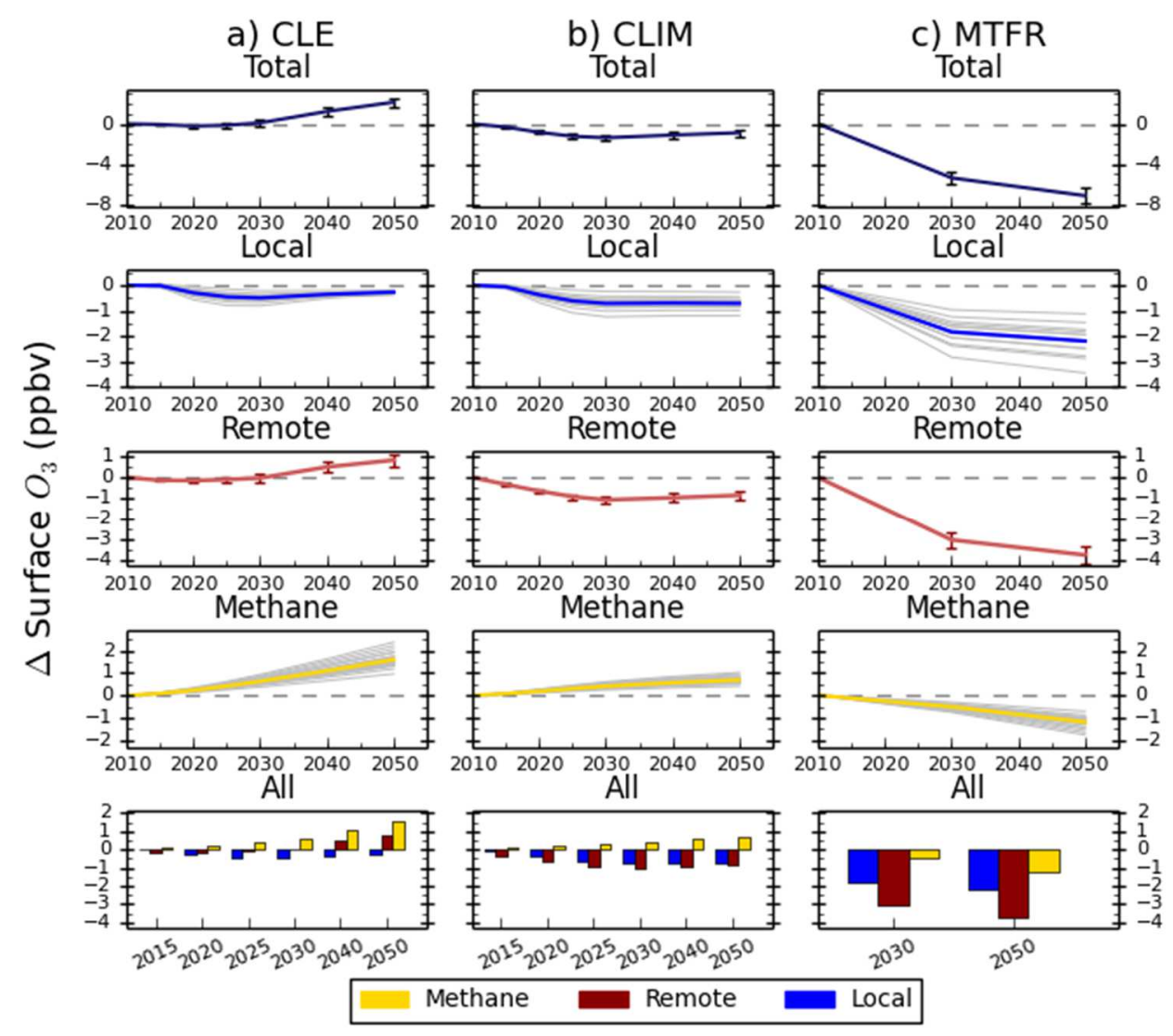

Figure S7: Same as Fig. S6 but for East Asia

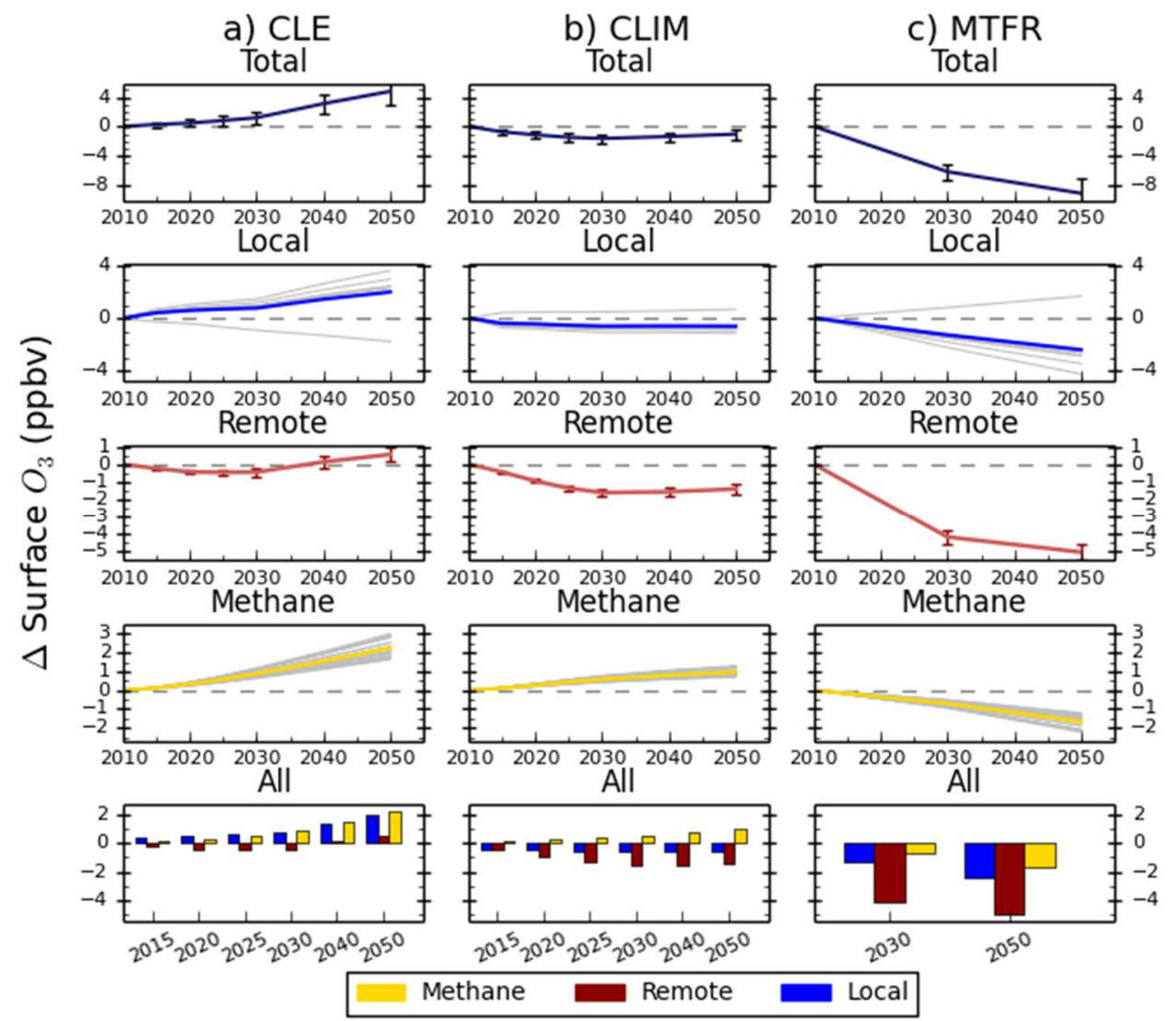

Figure S8: Same as Fig. S6 but for Middle East 


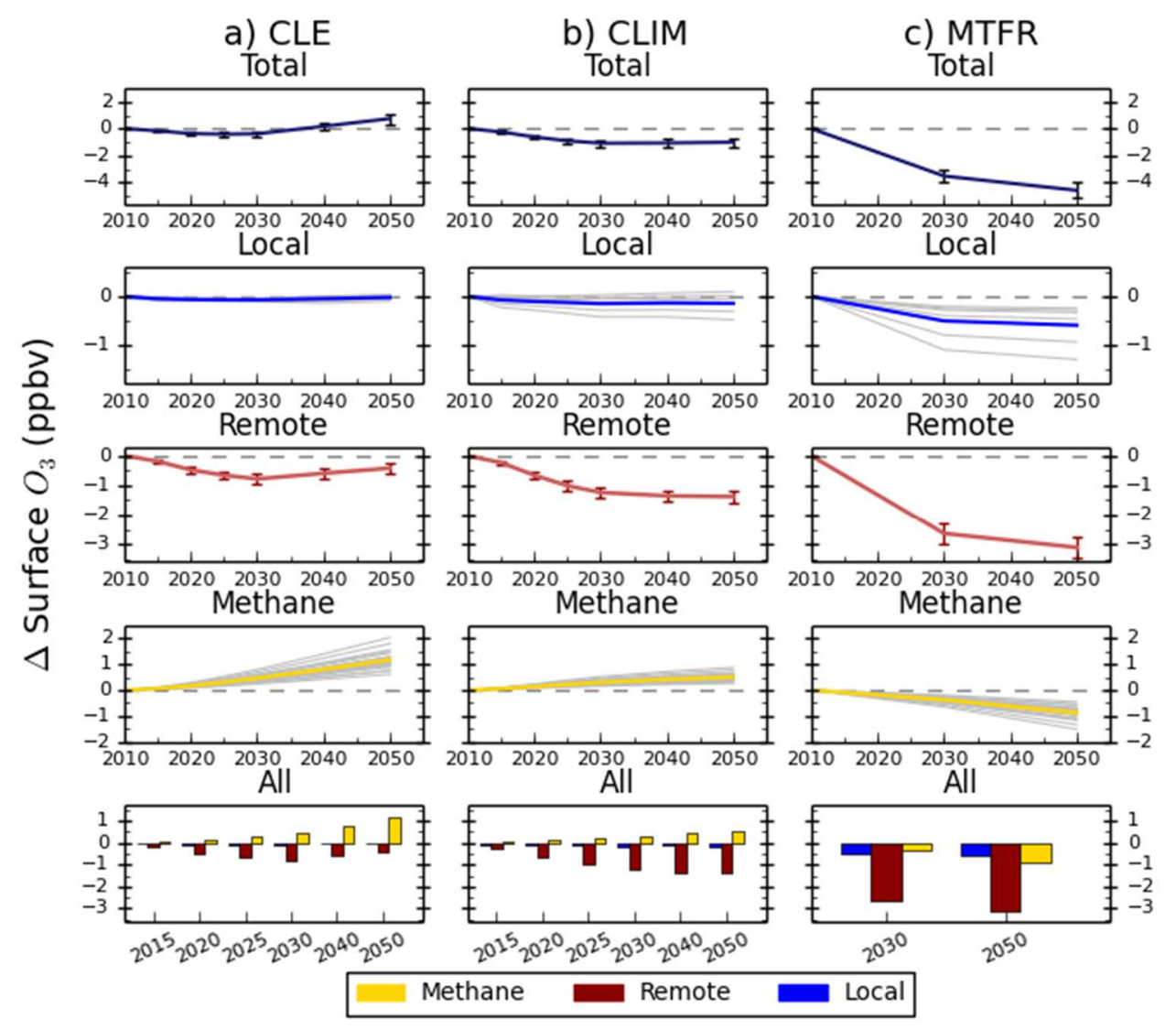

Figure S9: Same as Fig. S6 but for Russia Belarus and Ukraine

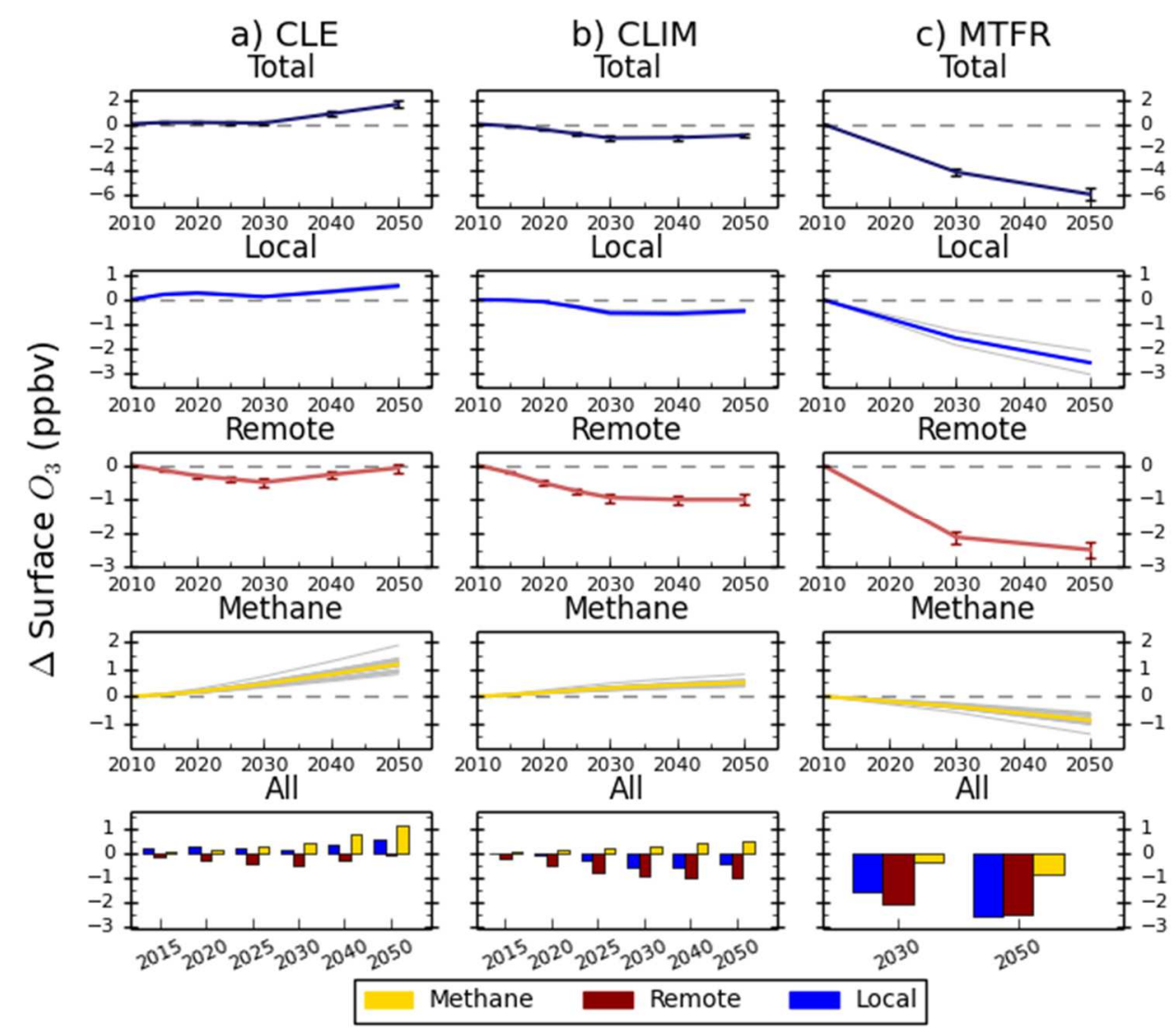

Figure S10: Same as Fig. S6 but for Central America 


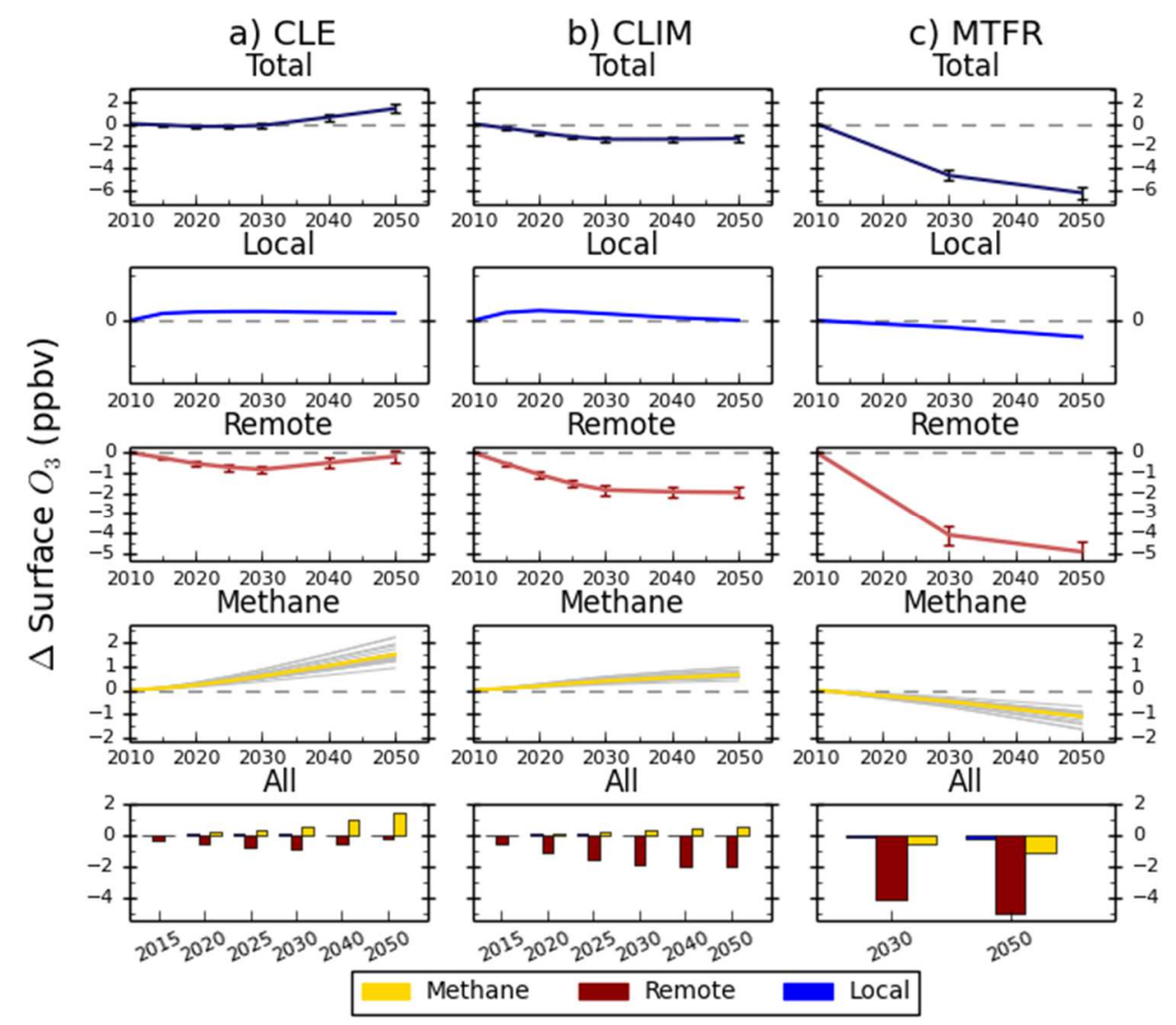

Figure S11: Same as Fig. S6 but for Central Asia

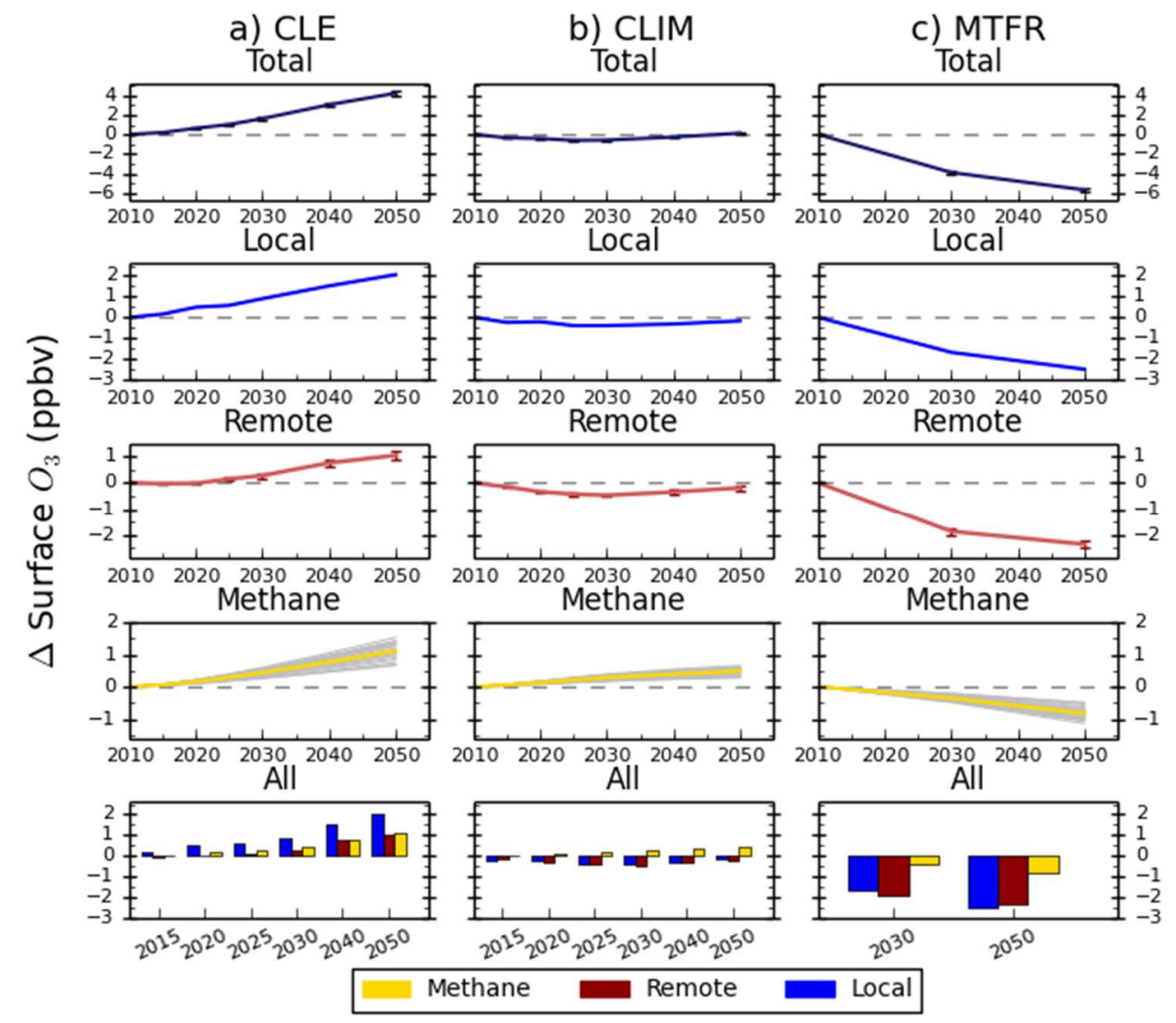

Figure S12: Same as Fig. S6 but for South East Asia 


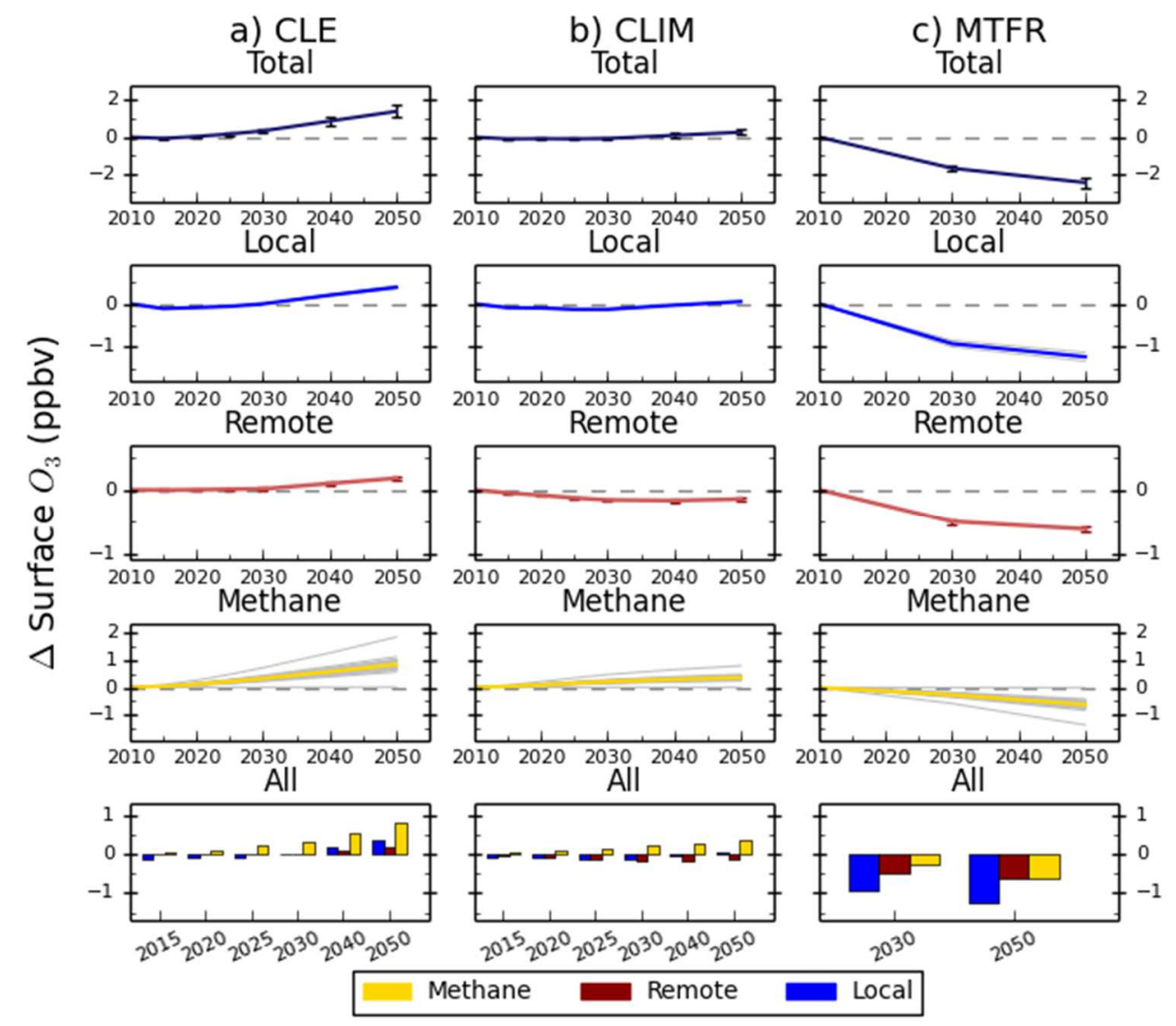

Figure S13: Same as Fig. S6 but for South America

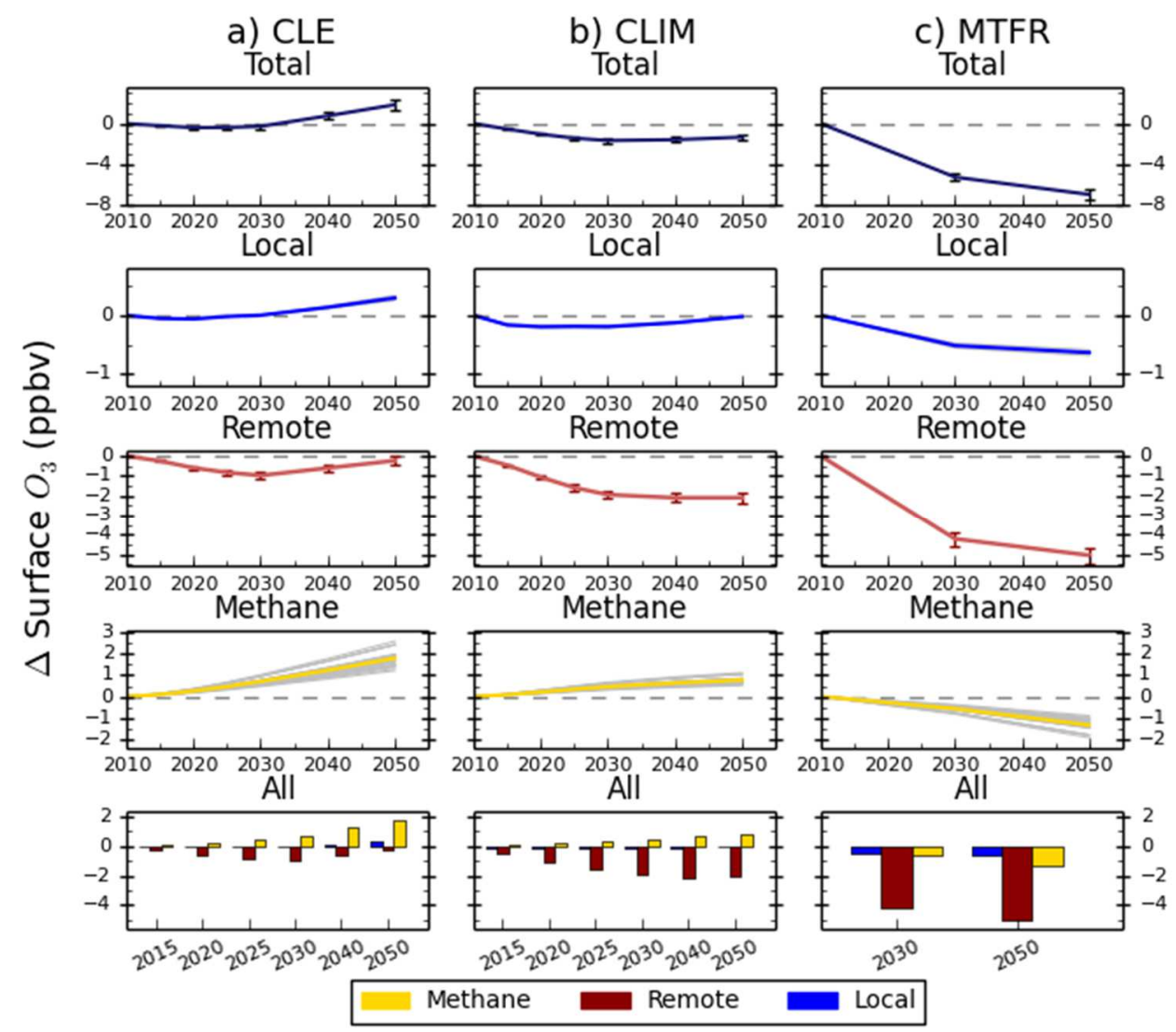

Figure S14: Same as Fig. S6 but for North Africa 


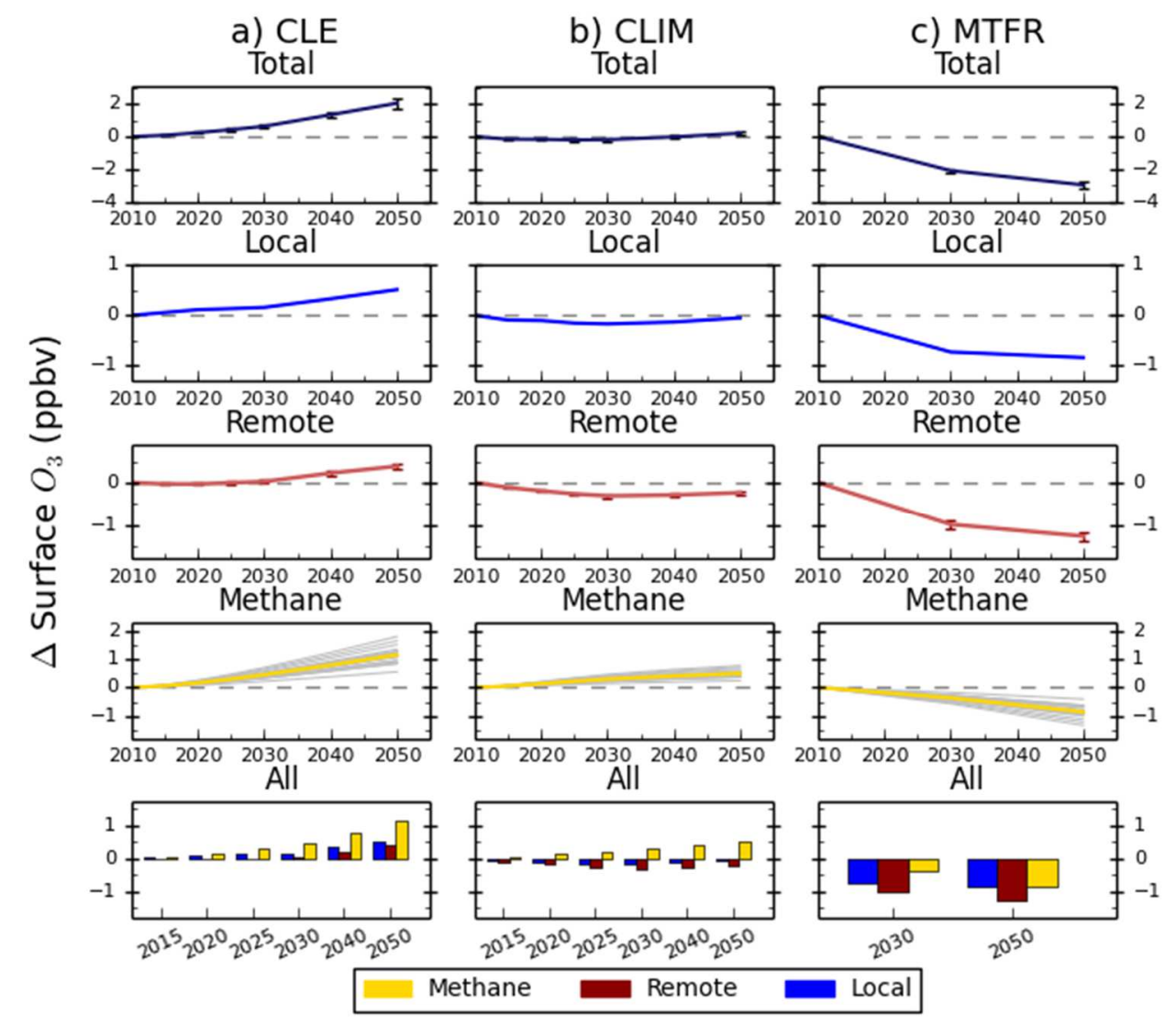

Figure S15: Same as Fig. S6 but for Southern (sub-Saharan) Africa

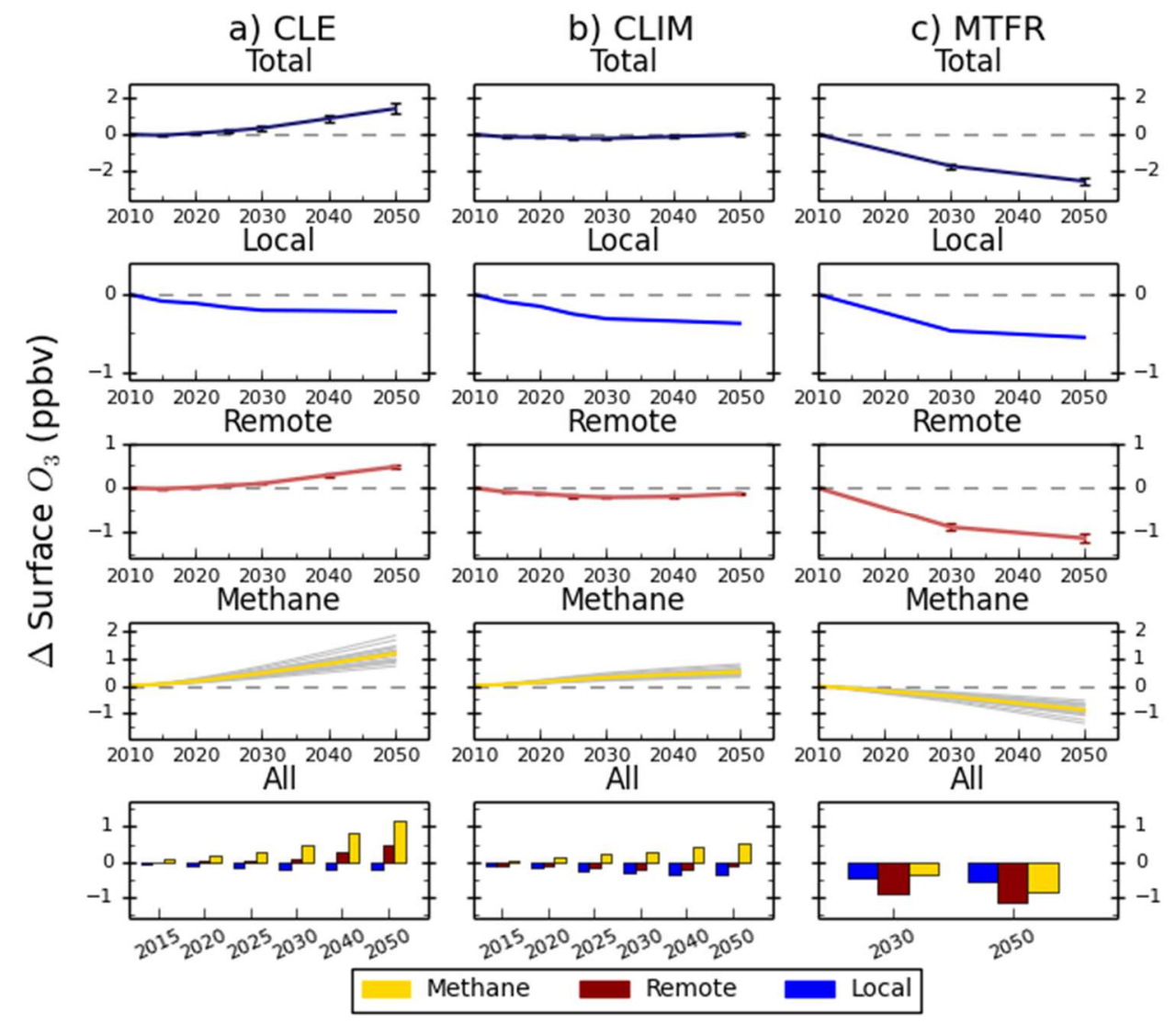

Figure S16: Same as Fig. S6 but for Pacific Australia and New Zealand 


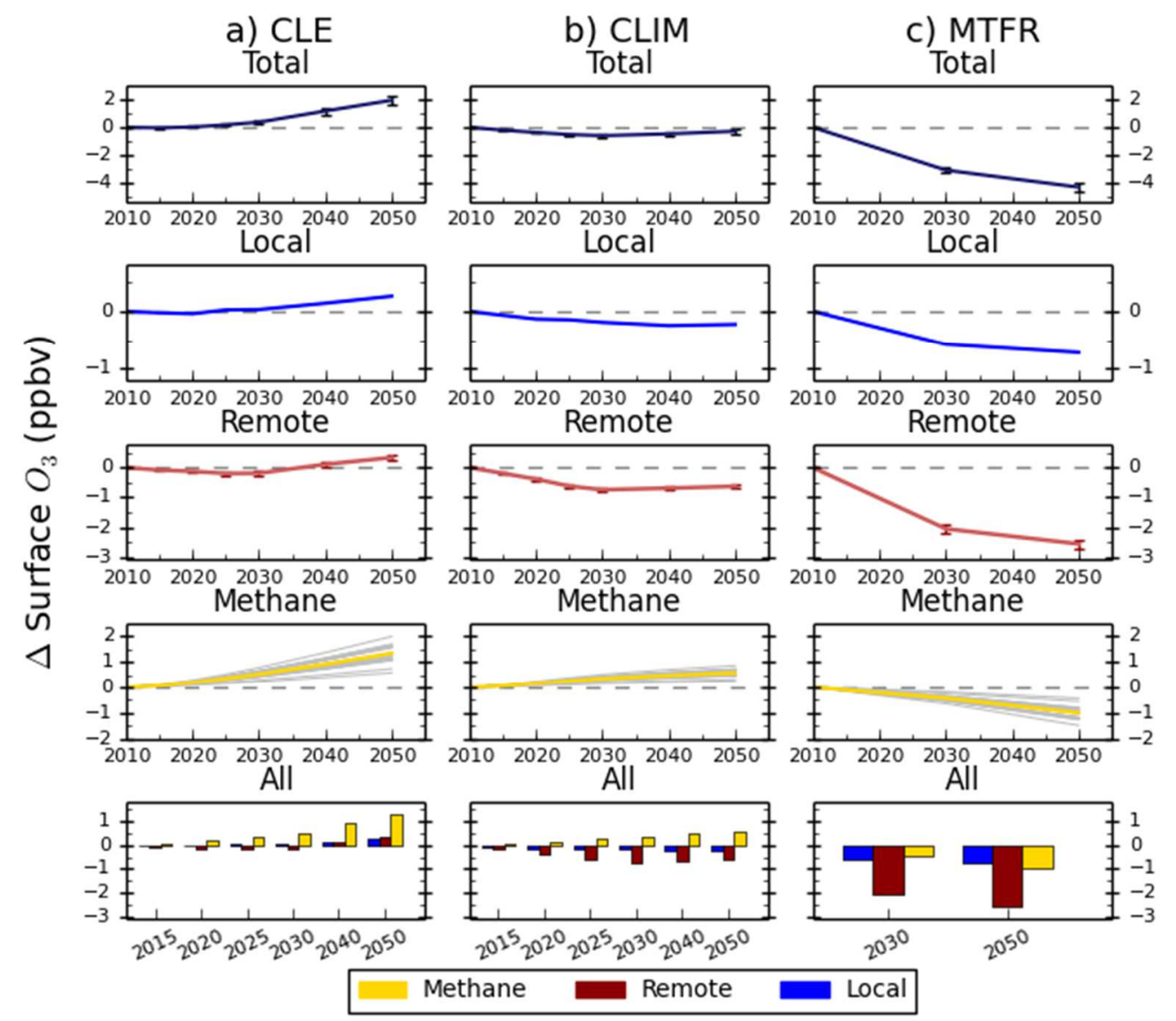

Figure S17: Same as Fig. S6 but for Ocean Regions 